\title{
PROBLEMS OF INSULATION DIAGNOSTICS OF POWER EQUIPMENT BY THE METHOD OF PARTIAL DISCHARGES
}

\author{
A.E. Usachev ${ }^{1, *}$, and A.Yu. Kubarev ${ }^{1}$ \\ ${ }^{1}$ Kazan State Power Engineering University, Kazan, Russia
}

\begin{abstract}
Target. High information value and reliability of diagnostics of isolation by partial discharges. Methods. Simulation of PD in Multisim and calculation of electrical fields of insulation defects by finite element method in the program ComSol. Results. A strong influence of the power supply on the value of voltage surge in case of emergency has been established. The method of adjustment of this influence is proposed. It was found that the oscillatory form of the PD is mainly determined not by the parameters of the insulation defect, but by the parameters of the energy object in which this defect is observed. Method for separation of aperiodic signal of PD from oscillatory component of system transient process in PD is proposed. It is shown that in operating turbogenerators and power transformers the unambiguous connection between the phase of PD occurrence and the voltage of PD occurrence is broken. Method of determination of PD occurrence place in slot part of turbogenerators is proposed. It is shown that in order to increase the reliability of comparing PD in polymer insulation of power cables of different voltage classes and geometric dimensions, when calibrating the measuring system, it is necessary to take into account the speed of propagation of the PD pulse through the cable and the capacity of the cable length unit. The concept of a corrective coefficient for calibration has been introduced and a method for determining it has been proposed. Conclusions. Taking into account the differences in PD parameters in real objects of the electric power industry, proposed in the article, from the parameters obtained in the standard laboratory model of the PD should increase the information and reliability of the PD method, as well as make it possible to conduct a comparative analysis of the results obtained under various observation conditions.
\end{abstract}

\section{Introduction}

The main reason for the irreversible deterioration of insulation (aging) properties under the influence of electric fields are electrical discharges in insulation defects, which bypass part of the insulation and are called partial discharges (PD) [1]. Standards for measuring PD parameters and their interpretation have been developed [2-4]. Tens of thousands of specialists in the world deal with insulation diagnostics, studying the characteristics of PD in insulation (PD method), in order to predict the time to the occurrence of an emergency due to breakdown of the insulation and closure of the current-carrying phase of the equipment to the ground. Extensive experience and an array of experimental data on PD in various energy facilities have been accumulated $[5-8,27,28]$. At the same time, when diagnosing turbine generators (TG) operating in the network, leading IRIS specialists, based on almost half a century of experience in monitoring the state of TG, abandoned the main characteristic of a single PD - an apparent charge, and leave only the magnitude of the voltage throw and the phase angle of the occurrence of PD. Based on almost a million TG surveys, they have developed criteria for processing a large array of single PD that can be used to predict the reliability of the TG.
These are largely empirical criteria that are weakly associated with isolation defects [9.10]. The proposed explanations of the patterns of the distribution of phase angles of the occurrence of PD often contradict the theory of breakdown of gas gaps. According to such operations, electric discharge can occur at zero potential difference due to the "adhesion of avalanche electrons" to the walls of the gas connection. Such models directly contradict both the theory of gas discharges and numerous laboratory studies [11-15]. In laboratory conditions, tens of thousands of experiments were conducted to break through gas gaps of various sizes and a fairly reliable link was established between the breakdown voltage and the value of the apparent charge with the size of the discharge gap [16-18].

In the isolation of real objects, PD is often observed in the form of oscillations. How to measure the main characteristic of the PD - the voltage throw in the TG insulation defect in this form of discharge? There is no definite answer to this question and there are several options for measuring this value. In laboratory conditions, PD is observed mainly in the form of a single aperiodic pulse and it is necessary to have fast-acting recorders to measure the amplitude of the PD. With PD in TG, the amplitude of the first half-period of the oscillating PD is in many cases less than the amplitude

\footnotetext{
* Corresponding author: usachev.ae@ @,kgeu.ru
} 
of the second half-period, which is poorly correlated with the discharge model in multilayer insulation. The same pattern of PD is observed in power transformers operating in the network.

The second main characteristic of the PD, unambiguously associated with the size of the defect, namely the voltage of the occurrence of PD in the operating transformers and $\mathrm{TG}$, is also not unambiguously determined. If with small sizes of the observation object in the laboratory, the voltage at all points of the high-voltage electrode is the same, then when connecting the windings of three-phase TG and power transformers (PT) into a "star" or "triangle," the amplitude of sinusoidal oscillations in different parts of the winding varies from zero to amplitude phase or interphase value. If it is not known in which part of the winding PD occurs, and this is the case in the vast majority of equipment, then the concept of the phase of the occurrence of PD loses the meaning of the occurrence voltage and is not related to the size of the defects. It is probably this circumstance that leads to the loss of the physical meaning of the pictures of amplitude-phase diagrams in TG and PT and the emergence of various interpretations of the measurement results. This is not observed if PD measurements are made on TG and PT, which are disconnected from the network, and the test voltage is supplied to the windings from a separate test transformer. In this case, the voltage at all sections of the current-carrying parts of the phases is the same and the unambiguous correspondence between the phase of occurrence of PD and the voltage is restored, which makes it possible to evaluate the amount of defects. It should be noted that the PD occurrence voltage can be affected by residual ion charges in the defect cavity if there are electronegative gases in the cavity.

Another feature of recording PD in operating TG and PT is that PD occur in the power supply. In model [11], it is assumed that during the time of the front of the PD pulse, the amount of charge on the observation object does not change, which leads to a decrease in voltage when shunting a part of the insulation and an increase in its capacity. This assumption is equivalent to no connection between the object and the PD and the power supply. If there is a power supply, then at low charge time constants, i.e. at low active resistance or inductance, the PD may not be observed at all in the measuring circuit through the separation capacitor. In the operating TG, each section of the slot part of the winding generates electromotive force (EMF), which reduces the value of the voltage surge at PD. If the TG is disconnected from the network, then the amount of voltage surge should increase.

From the above it is quite obvious that the conditions for observing the breakdown of air gaps in laboratory conditions and in real objects of the electric power industry can vary significantly. A study of such differences and problems of PD observation is given in this work.

\section{Partial discharges in operating turbine generators}

In the TG (on-line mode) operating in the network, PD occurs in the EMF source, which can reduce the amount of voltage surge. The voltage at different insulation points of the conductive rods of the TG phase is different, and the phase length is much longer than the wavelength corresponding to the PD. To take these factors into account, the PD was simulated for isolation of the hydrogen forced cooling turbine generator (TVF-60-2) brand turbogenerator in the Multisim software environment [19].

Each of the two parallel branches of the TG phase (for example, for phase $\mathrm{A} 1 \mathrm{C}_{1}, 2 \mathrm{C}_{1}$ fig.1) consists of 24 series-connected conductive rods. In each of the 72 stator slots there are 2 rods (fig.2), which are isolated from each other by a slot (body) mycanite insulation with a thickness of $4 \mathrm{~mm}$ and a middle $4 \mathrm{~mm}$ glasstextile gasket with a two-sided copper foil coating. Partial discharges in the slot portion may occur between the conductor and the stator housing. The voltage on the conductive parts of the TG operating in the network varies from 0 at the common phase connection point to an amplitude value of $8570 \mathrm{~V}$ at the phase output. In the slot portion of the $\mathrm{TG}$, the voltage between the conductive rod and the housing differs by $357 \mathrm{~V}$ at the beginning and end of each of the 24 phase rods. In the frontal parts of the TG, the EMF is not induced, and the voltage difference is determined by a voltage drop when current flows through the active resistance of the section. It does not exceed $0.5 \mathrm{~V}$, that is, it can be considered that all sections of the same conductive rod are under the same voltage. From such a TG design, it becomes clear that in different parts of the TG phase, the insulation is under various stresses. Connection diagram of stator winding rods of THF-60-2 turbine generator is shown in fig. 1

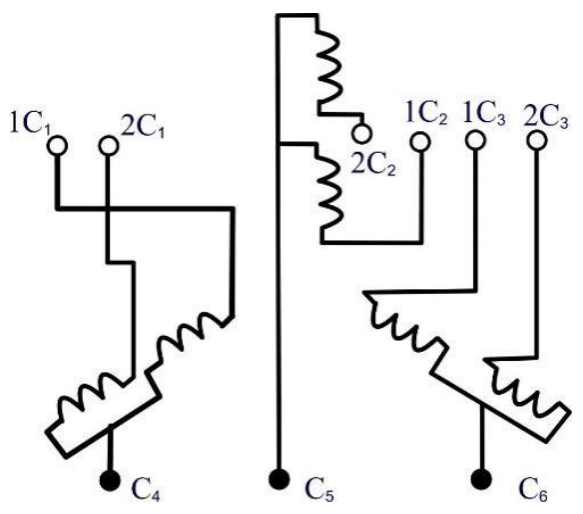

Fig. 1. Connection diagram of stator winding rods of TG TVF$60-21 \mathrm{C}_{1}, 2 \mathrm{C}_{1}\left(1 \mathrm{C}_{2}, 2 \mathrm{C}_{2}, 1 \mathrm{C}_{3}, 2 \mathrm{C}_{3}\right)$ - phase outputs branches of winding $\mathrm{A}$ (B. C); $\mathrm{C}_{4}, \mathrm{C}_{5}, \mathrm{C}_{6}$ - general (zero) outputs of respective phases.

Section of one stator slot with current-conducting rods and insulation is shown in fig. 2. 


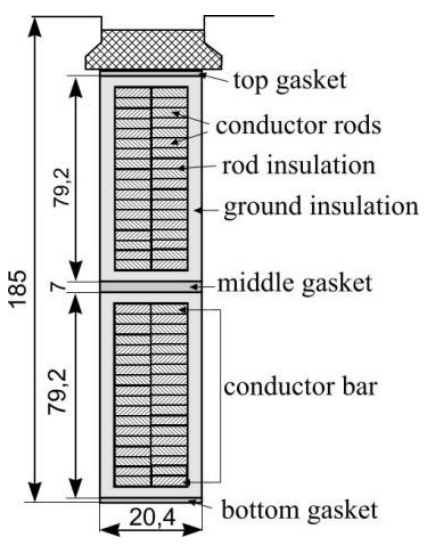

Fig. 2. Section of stator slot with current-conducting bars.

With this design of the slot part of the TG, the resulting partial discharges occur, in fact, in a uniform electric field. To assess the effect of the power supply on the amount of voltage surge during the PD, modeling was carried out in Multisim. In the model, the design, consisting of a conductive insulation rod and a TG housing, was in the form of a flat capacitor with a height equal to the thickness of the insulation. Deviations from the uniform field near the corner parts of the rods were not considered. Diagram of replacement of insulation with defect in accordance with models [1.11.12] is shown in fig. 3 .

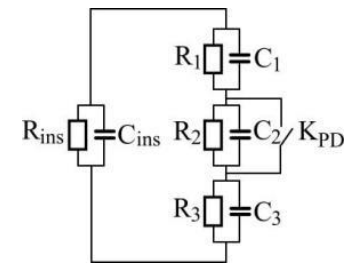

Fig. 3. Insulation replacement scheme with defect.

The insulation of the rod (fig. 3) can be represented as a column with a defect $\left(R_{i}, C_{i}\right)$ and the rest of the defect-free part $\left(R_{i n s}, C_{i n s}\right)$. The defect $\left(R_{2}, C_{2}\right)$ could be located at an arbitrary distance between the currentcarrying part and the TG body, which was modeled by values $\left(R_{1}, C_{1}\right.$ and $\left.R_{3}, C_{3}\right)$. The $\mathrm{PD}$ event was simulated by closing the PD key. The measurement of PD values in TG is usually carried out according to a circuit with a separating capacitor. If the TG is disconnected from the network and isolation is diagnosed from an external, independent power supply, then the replacement circuit has the form [19]:

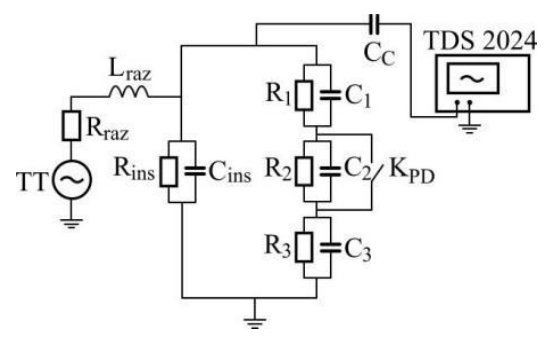

Fig. 4. Model in Multisim of PD registration in TG (off-line) powered by test transformer.
In Figure 4, TDS 2024 is a four-channel oscilloscope; TT - test transformer (TT); $\mathrm{C}_{\mathrm{C}}$-connecting capacitor; $L_{r a z}$ and $R_{r a z}$ - inductance and active resistance in the circuit section from TT to the defect site in the slot rod insulation ("isolation"); $K_{p d}$ - key (switch) simulating PD in defect. The TT is connected to one of the phase leads of the winding (fig. 1). This TG isolation diagnostic scheme is used when it is disconnected from the network (off-line mode). In this mode, the EMF is not induced and in all parts of the stator winding the voltage is the same and equal to the TT voltage. When measuring PD according to this scheme, the phase of the voltage at which PD occurs is uniquely associated with the voltage of PD occurrence. Since in such a circuit a PD can occur in a defect located in any part of the winding, $L_{r a z}$ can vary from zero if the defect is at the phase terminal to the inductance of the entire leg of the TG phase $(\sim 1.2 \mathrm{H})$ if the defect is located at the zero (general) terminal. PD oscillograms during simulation according to scheme 4 are shown in fig. 5 at different $L_{r a z}$ values.

With $L_{r a z}=0$, which corresponds to the location of the defect directly at the phase output, the PD signal is absent. Inductance of one winding rod $\sim 120 \mu \mathrm{H}$, i.e. PD in the same size defect, but located at the end of the first rod, will be observed. The oscillatory form of this PD has the form not usual for a transition process. Amplitude of the first minimum, from which voltage throw at PD is determined, is less than amplitude of the second maximum. This form of PD is often observed in $\mathrm{TG}$, which gave rise to various assumptions how to correctly determine the amplitude of the voltage throw at PD. As will be shown below, they are not all correct. In figure 5.b, the amplitude of the 1st minimum has increased and is almost the same as that of the 2nd maximum, the shape of the PD still has an oscillatory appearance, but the half-width of the 1 st minimum is different from the 2nd maximum. This picture corresponds to the position of the defect at the end of the second rod from the phase output. With a further increase in $L_{r a z}$, which is equivalent to shifting the position of the defect to the general output, the amplitude of the 1st minimum increases, and the signal PD at the same time scale turns into aperiodic. If you change the scale of the oscilloscope scan over time, then the oscillatory process is clearly visible, but the frequency of this process is determined by the values of $L_{r a z}$ and $C_{i n z}$. The size of the defect $\left(R_{2}, C_{2}\right)$ is practically unaffected by the frequency of the transient process. Since in such a simulation the defect value remained constant, it can be concluded that the main measured value at the PD - the voltage throw depends on the practically uncontrolled parameter of the experiments - the remoteness of the power source. If the inductance between the power source and the PD test object is small, the assumption that the amount of charge on the insulation tank remains constant during the PD ceases to be carried out due to the recharging process when the voltage decreases (at the PD). The voltage throw itself, which would be observed in the absence of feed from the source, is an aperiodic signal of the type fig. 5.d. In TG, the form of PD in many cases has the form of fig.5a, which indicates the superposition of the aperiodic signal of PD and the 

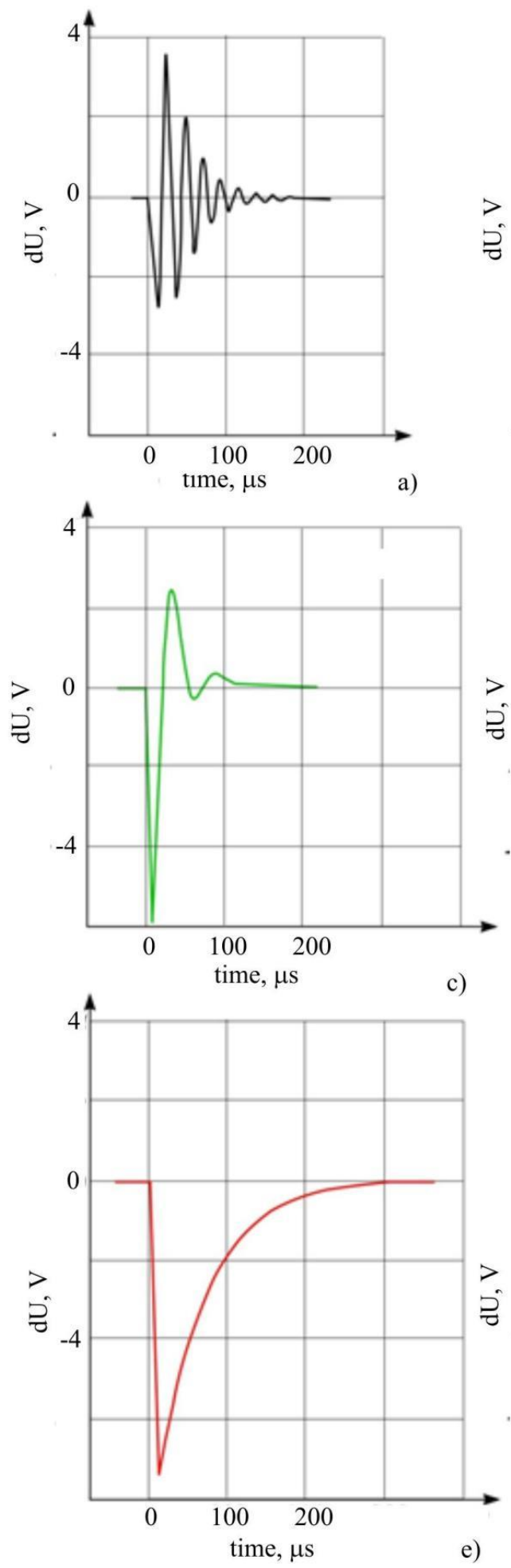

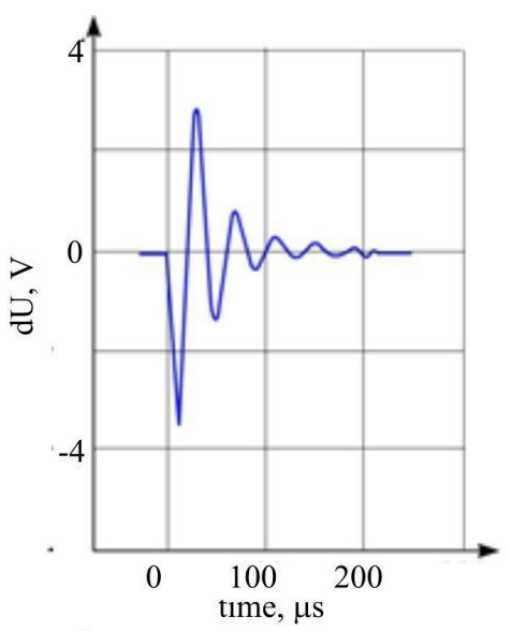

b)

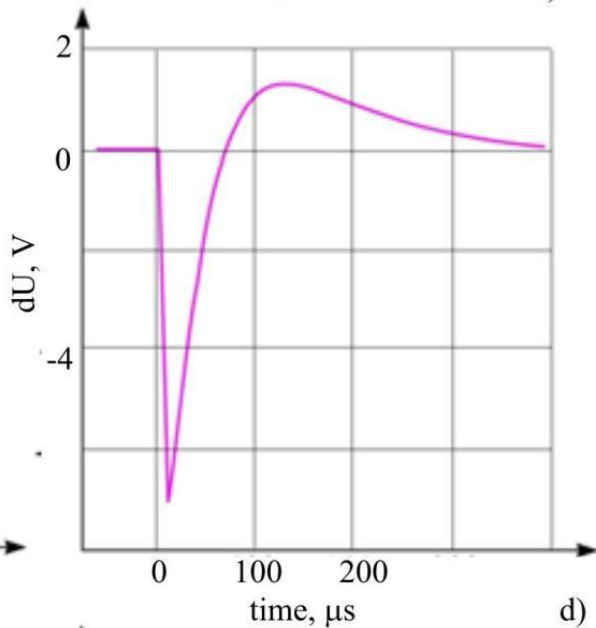

d)

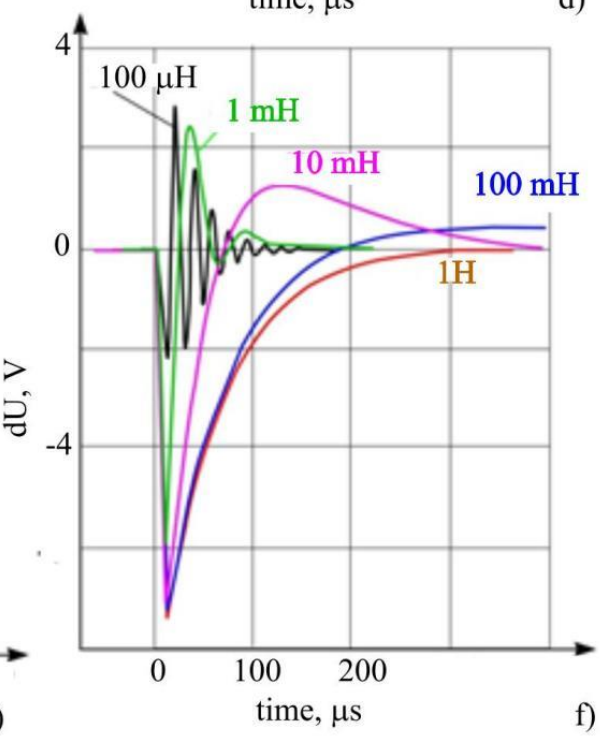

Fig. 5. Oscillograms of model PD at different $L_{r a z}$ and the same amount of defect in insulation, where a) $100 \mu \mathrm{H}$, b) $230 \mu \mathrm{H}$, c) $1 \mathrm{mH}$, d) $10 \mathrm{mH}$, (e) $1 \mu \mathrm{H}$ and f) at different $L_{r a z}$.

periodic oscillatory transition process. In this case, the extraction of the real amplitude of the PD corresponding to the absence of feed from the source is possible as follows [19].

1. Value of voltage surge $d U(L)$ is measured at value of inductance to source unknown in experiment $(L=$ $\left.L_{r a z}\right)$.
2. The inductance to the source $\left(L_{r a z}\right)$ is determined from the calculated capacitance of the rod insulation $\left(C_{i n s}\right)$ and the measured frequency of oscillation of the PD pulse.

3. The least squares method is the PD simulation approximation function for different values of $L=L_{r a z}$ and the obtained formula determines the value of $d U(L)$.

4. Correction factor is determined 


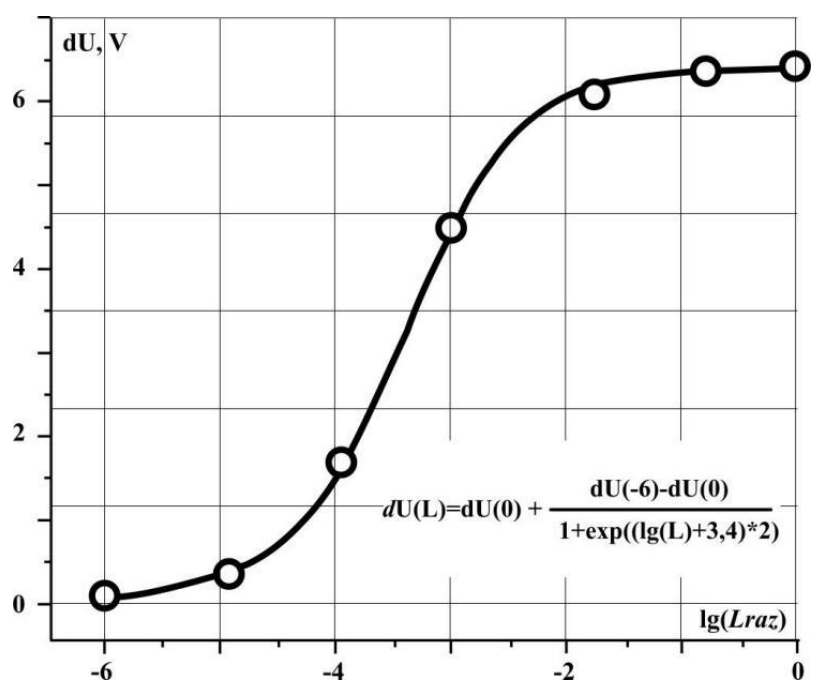

Fig. 6. Dependence of PD amplitude in diagram (fig.4) on inductance between voltage source and object with PD.

In [19], it has been shown that the change in the voltage at $\mathrm{PD}$ over the duration of the leading edge affects section 1 of the TG winding rod. The combination of the measurement of the apparent charge value according to the method [19] and the use of experimental data on the voltage of the occurrence of PD in various gases [15-18] made it possible to propose, in addition to traditional methods of presentation, a new representation of the measurement results of $\mathrm{PD}$ in turbogenerators $[20, .21]$. This representation is based on the assumption that PD in a separate gas connection occurs after recombination of the volume charge remaining after the previous PD. In this approach, measuring the phase of the occurrence of PD allows you to determine the location of the defect with accuracy to the rod. The result of processing of PD measurements according to this method is shown in fig. 7.
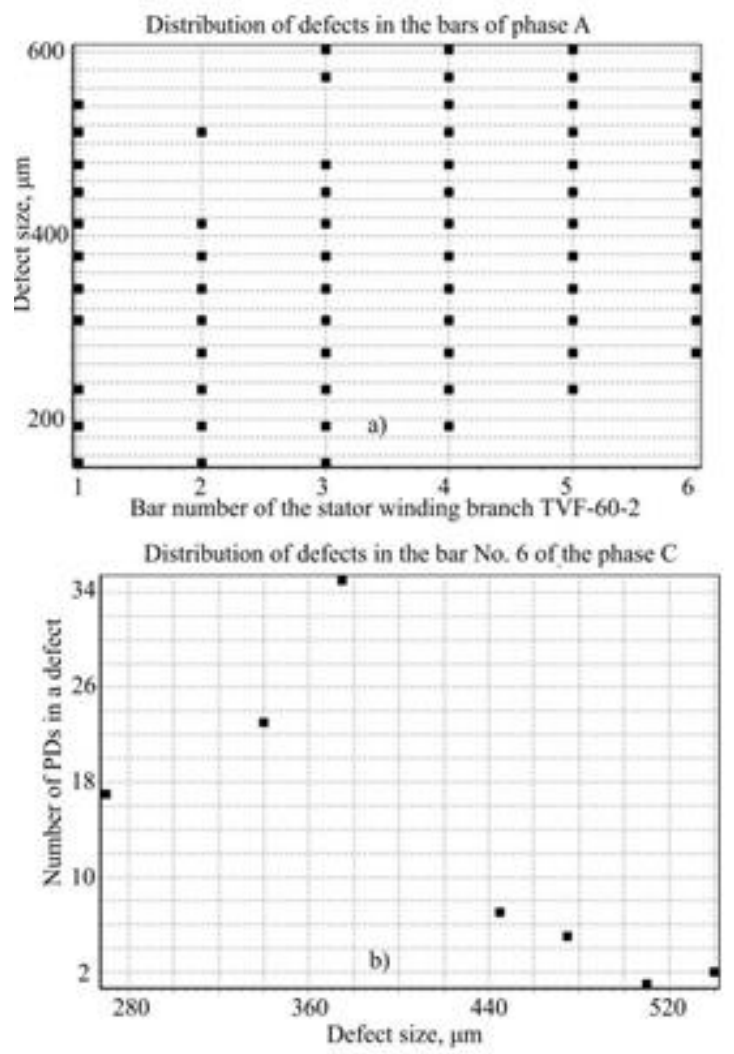

Fig. 7. Presentation of PD measurement results in the turbine generator TVF-60-2: a) distribution of defects by bars; b) number of PD in defects of one bar.

This view provides a better picture of the state of the insulation and the presence of defects in it. A further development of this representation could be the numerical solution of Poisson equations in gas pores, that is, the accounting of residual volumetric charges from previous $\mathrm{PD}$.

In power transformers operating in the network, the general picture of the occurrence of PD is similar to the situation in TG due to the difference in electric field strength in different parts of the PT windings. However, there are significant differences:

1. Windings have a complex shape (coil, multilayer, compensating and red)

2. Non-uniform insulation (paper, oil, barriers)

3. Presence of capacitive connection between winding turns and difference of longitudinal winding capacity inside the layer and between layers.

At the same time, at the frequencies of the PD winding, it is fashionable to consider the analogues of long lines, i.e., the voltage throw in the PD affects only a part of the winding and the calculation of the apparent charge is even more complex, the number in the turbogenerators.

\section{Partial discharges in cross-linked polyethylene cables}

Up to $85 \%$ of emergency situations in cable transmission lines (CL) are associated with the development of defects in insulation, leading to its breakdown. The main 
reason for the deterioration of the insulation properties are microscopic holes of the insulation part known as partial discharges. A large number of works are devoted to PD diagnostics [22-26]. Unlike power transformers (PT) and turbine generators operating in the network, in the CL the power source, which is the PT at the substation, is clearly defined. The length of the CL is usually not more than a few kilometers, which is much less than the wavelength of the industrial frequency, i.e. the voltage at all points of the CL is the same with good accuracy. In this case, the phase of PD occurrence is uniquely related to the voltage on the central core of the $\mathrm{CL}$ at the place of PD occurrence. Our analysis of the values of induced stresses on the $C L$ screen with a complete transposition cycle of the screens allows us to argue that even in the middle of the transposition segment under the normal mode of operation of the CL, this voltage does not exceed several tens of volts and can be neglected when modeling fields inside insulation defects. Unfortunately, the field in the cable insulation is not uniform. This leads to the fact that only by the voltage of the occurrence of PD, it is not possible to calculate the size of the defect. Using a method similar to that developed for $\mathrm{TG}$, this can be done if you determine the amount of apparent charge of the PD.

Among the various methods for monitoring the state of insulation, the CL during operation received a method for measuring PD using high-frequency current transformers of the RFCT type, which are put into the circuit for connecting CL screens during phase transposition to non-linear surge limiters (NSL). In order to assess the influence of the distance from the substation to the PD origin, a simulation was carried out in Multisim according to the diagram of fig.8.

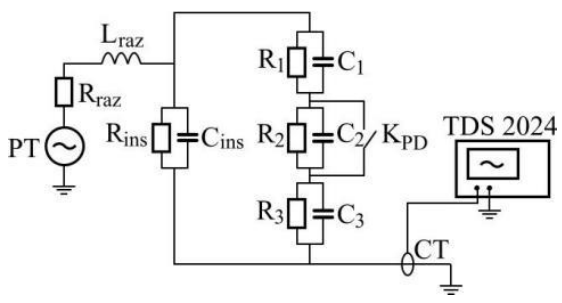

Fig. 8. Scheme of PD registration with CT (RFCT) in Multisim.

Symbols in fig. 8 correspond to symbols in fig. 4 . The inductance $L_{r a z}$ was taken as the inductance of the CL from the PT substation to the place of occurrence of the PD. The rate of electromagnetic wave propagation in the $\mathrm{CL}$ with insulation from cross-linked polyethylene (CLP) is about $200 \mathrm{~m} / \mu \mathrm{s}$. During the PD front, the PD momentum manages to spread to a distance of $2 \mathrm{~m}$ in each direction. If we consider that the change in voltage at the pulse front is a linear function of time, then the region of the $\mathrm{CL}$ at which the change in voltage will occur will be no more than $2 \mathrm{~m}$. The capacity of this particular region should be used to calculate the amount of apparent charge. Dependence of voltage surge for diagram fig. 8 . is shown in fig. 9 .

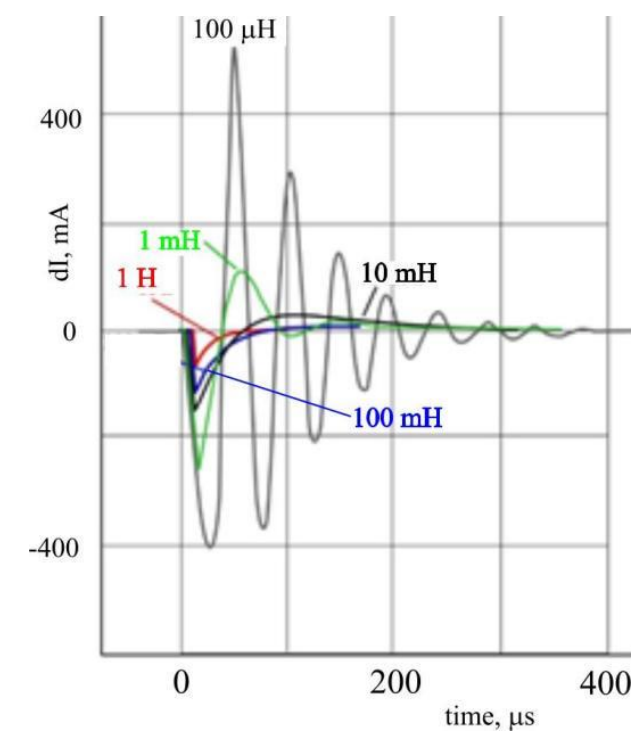

Fig. 9. PD form in fig. 8 scheme at different $L_{r a z}$ values.

In this scheme, as in TG, there is a clear dependence of the value of the voltage throw on the distance to the power supply $\left(L_{r a z}\right)$. The closer the power supply (PT substation), the greater the throw amount. All PD of fig.9 correspond to the same defect size. As in TG, the main measured value at PD - the voltage throw depends on the inductance of the CL from the PT substation to the PD location. For cable lines, this value can be determined by the distance to the PD location, which is measured simultaneously with the voltage surge. The distance is determined by the difference in the arrival times of the straight and reflected from inhomogeneities (usually the end of the line or connecting couplings) and the propagation rate of the PD pulse. Then it is possible to enter a correction factor for the amount of voltage surge, which depends on the distance to the substation $\left(L_{r a z}\right)$. The amplitude attenuation factor can be neglected due to the relatively small lengths of cable lines and the absence of corona in normal operation modes.

Calibration of the measuring system by introducing a voltage pulse from the calibration generator into the CL shall take this into account. Another feature of calibrating the system for measuring PD in the CL is that the linear capacity of the CL with a different section of the conductive core can differ many times $(120 \div 390$ $\mathrm{nF} / \mathrm{km})$.

The calibration generator shall have a tunable capacitance corresponding to the capacitance of the CL. Taking into account the propagation rate of the PD pulse leads to the fact that the capacity of the pulse generator in the calibration generator should be equal to the capacity of $\sim 2 \mathrm{~m} \mathrm{CL}$, which is $240 \div 780 \mathrm{pF}$. The use of pulse voltage generator (PVG) for calibration with a capacity of $100 \mathrm{pF}$ leads to overstated values of the apparent charge at remote from the PD substation.

\section{Conclusion}

When interpreting the results of PD measurements in turbogenerators, power transformers and power transmission cable lines, it is necessary to take into 
account the influence of the power source on the measured parameters of partial discharges. In the turbine generators and power transformers operating in the network, the voltage in various parts of the winding is measured from zero to the amplitude value of the interphase voltage, which disrupts the unambiguous connection of the PD phase and the occurrence voltage. Taking into account the differences in PD parameters in real objects of the electric power industry, proposed in the article, from the parameters obtained in the standard laboratory model of the PD should increase the information and reliability of the PD method, as well as make it possible to conduct a comparative analysis of the results obtained under various observation conditions.

\section{References}

1. G.S. Kuchinsky, Partial discharges in high-voltage structures (L.: Energy, 1979)

2. Yu.V. Sharov, G.F. Binko, V.V. Belyakov, Digitalization of current state control and predictive diagnostics of turbogenerators at power plants, Digital electric power industry, Unified network energy, 6 (69), 19-30 (2019)

3. J.F. Lyles, G.C. Stone, M. Kurtz, Experience with PDA Diagnostic Testing on Hydraulic Generators, IEEE Trans EC, 824 (December 1988)

4. Li. Shengtao, Li. Jianying, Condition monitoring and diagnosis of power equipment: review and prospective, High Volt., 2 (2), 82-91 (2017)

5. S. Grubic, J.M. Aller, B. Lu, T.G. Habetler, A Survey on Testing and Monitoring Methods for Stator Insulation Systems of Low-Voltage Induction Machines Focusing on Turn Insulation Problems, IEEE Trans. Indus. Elect., 55 (12), 4127-4136 (2008)

6. Y.J. Kim, H.D. Kim, Novel partial discharge monitoring scheme for rotating machine, The 19th Int. Symp. HV Eng., Pilsen (2015)

7. G. Berg, E. Eberg, S. Hvidsten, Partial Discharge Characterisation of Stator Windings Taken from a 50-year-old Norwegian Hydrogenerator, Electrical Insulation Conference (EIC) 2019 IEEE, 168-171 (2019)

8. R.P. Nair, S.B. Vishwanath, Analysis of partial discharge sources in stator insulation system using variable excitation frequency, IET Sci. Meas. Technol., 13 (6), 922-930 (2019)

9. B.S. Reddy, A.R. Verma, Novel technique for electric stress reduction across ceramic disc insulators used in UHV AC and DC transmission systems, Appl. Energy, 185 (2), 1724-1731 (2017)

10. G.C. Stone, PD diagnostics and electrical equipment insulation condition assessment, IEEE Trans. Dielectr. Electr., 12 (5), 891-904 (2005)

11. V.P. Vdoviko, Partial discharges in the diagnosis of high-voltage equipment (Novosibirsk: Science, 155, 2007)
12. V.A. Rusov, Measurement of partial discharges in insulation of high-voltage electrical equipment (Yekaterinburg: UrGUPS, 2011)

13. G.A. Month, Laws of similarity in pulse gas discharges, UFN, Reviews of current issues, 176 (10), 1069-1091 (2006)

14. G. Paoletti, A. Golubev, Partial Discharge Theory and Technologies related to Medium Voltage Electrical Equipment, IEEE-1999, 99-25 IEEE IAS 34th Annual Meeting, Phoenix, Arizona USA (3-7 October 1999)

15. F.R. Ismagilov, D.V. Maksudov, Mathematical modeling of the development of partial discharges in the process of dielectric aging, Bulletin of the Ufa State Aviation. those. un-ta., 15-3 (43) (2011)

16. H.A. Illias, G. Chen, P.L. Lewin, Modelling of partial discharge activity in different spherical cavity sizes and locations within a dielectric insulation material, Properties and applications of dielectric materials, ICPADM 2009, IEEE 9th int. Conf. 2009, 485-488 (2009)

17. A.F. Alexandrov, V.L. Bychkov, L.P. Grachev, I.I. Isakov. A.Yu. Lomteva, Air ionization in the nearcritical electric field, GTF, 76 (B.3), 38-43 (2006)

18. Yu.P. Reiser, Gas discharge physics (M: Science, 536, 1992)

19. A.M. Ba Boraik, A.E. Usachev, A.Yu. Kubarev, S.M. Margulis, Features of observing partial discharges in stators of turbine generators included in the network, News of higher educational institutions, Energy problems, 1 (3-4) (2017)

20. A.M. Ba Boraik, A.E. Usachev, A.Yu. Kubarev, Modeling the process of partial discharge in the computer environment, University news, Energy problems, 9-10 (2016)

21. A.M. Ba Boraik, A.E. Usachev, M. Ali Ziad, A New Proposed Method for Interpretation Online Partial Discharge Measurements Data of Turbine Generator, Journal of Electrical Engineering \& Technology, 15, 1301-1311 (2020) DOI: 10.1007/s42835-020-00414-9

22. A.Yu. Kubarev, A.Ye. Usachev, T.V. Lopukhova, Yu.G. Kubarev, Methods of detection of hazardous areas in the insulation of cable lines according to the characteristics of partial discharges, University news, Energy problems, 1-2, 79-83 (2012)

23. D.A. Polyakov, K.I. Nikitin, N.A. Tereshchenko, I.V. Komarov, U.V. Polyakova, Study of the dependence of partial discharge power on voltage in cables with insulation from cross-linked polyethylene, Omsk Scientific Bulletin, 1 (169), 3944 (2020) DOI: 10.25206/1813-8225-2020-169-3944

24. S.S. Refaat, M.A. Shams, A review of partial discharge detection, diagnosis techniques in high voltage power cables, 2018 IEEE 12th International Conference on Compatibility, Power Electronics 
and Power Engineering, CPE-POWERENG 2018, 1-5 (2018) DOI: 10.1109/CPE.2018.8372608

25. S.M. Korobeynikov, A.V. Ridel, D.A. Medvedev, et all, Registration and simulation of partial discharges in free bubbles at AC voltage, IEEE Transactions on Dielectrics and Electrical Insulation, 26 (4), 10351042 (2019) DOI: 10.1109/ TDEI.2019.007808

26. X. Chen, Y. Xu, X. Cao, Nonlinear time series analysis of partial discharges in electrical trees of XLPE cable insulation samples, IEEE Transactions on Dielectrics and Electrical Insulation, 21 (4), 1455-1461 (2014) DOI: 10.1109/ TDEI.2014.004307

27. D. Ivanov, M. Sadykov, A. Golenishchev-Kutuzov, , The application of the technology of sensor networks for the intellectualization of the overhead power transmission lines, E3S Web of Conferences 220,01071 (2020)

28. D. Ivanov, T. Galieva, M. Sadykov, A. Golenischev-Kutuzov, A. Naumov, Method for the diagnosis of high-voltage dielectric elements during operation based on dynamic registration of electromagnetic radiation, E3S Web of Conferences $216,01061(2020)$ 